International Journal of Engineering \& Technology, $7(2.13)(2018)$ 314-318
International Journal of Engineering \& Technology
SPC
Website: www.sciencepubco.com/index.php/IJET
Research Paper

\title{
Comparison of beta-kurtosis and kurtosis methods for troubleshooting the performance of a transmission vehicle using vibrating frequencies
}

\author{
Alireza Dadkhah Laleh ${ }^{1}$, Mirmohammad Ettefagh ${ }^{1}$, Reza Hasanezhad Qadim ${ }^{1}$ \\ ${ }^{1}$ Department of Mechanical Engineering, University of Tabriz, Tabriz, Iran \\ *Corresponding author E-mail: Alireza.dadkhahl@gmail.com
}

\begin{abstract}
One of the main methods in maintenance and repair is a preventive maintenance method that is often more effective. The requirements of this method are to monitor the performance of machinery during operation. One of the car's functions that is monitored in this way is its vibra-tions. In this paper, a mathematical model of vibration analysis of a passenger car gearbox is presented based on Beta-Kurtosis and Kurtosis methods. In the next step, the data and test settings for the gearbox are based on the accelerometer installation to record the vibrations of the gearbox. To verify the accuracy of the proposed method, the results of the vibrational analysis of the car gearbox in four modes of a healthy gearbox, defective gearbox in the shaft end bearing, gear shaft failure on the gear shaft, simultaneous failure of the bearing and gearbox on the gear shaft were compared. Also, the results are compared for both Kurtosis and Beta-Kurtosis methods. The results show that both of the proposed methods are very accurate in identifying faults in the gearbox and determining the type of fault.
\end{abstract}

Keywords: Gearbox; Troubleshooting; Beta-Kurtosis; Kurtosis; Vibrational Frequency Analysis.

\section{Introduction}

Today, with the growth of industry and the increasing use of industrial machinery, the maintenance of these machines and the evaluation of their operation during condition monitoring is of particular importance. As far as 5 to 12 percent of fixed capital in the industry is concerned with maintenance and repair costs (these costs also add product quality). The three main methods in maintenance and repair, the preventive maintenance method are most effective in most cases. The requirements of this method are to monitor the performance of machinery during operation. One of the car's functions that is monitored in this way is its vibrations. In 1989, McFadnan used an interpolation method for the mean time domain signal. [1] In a paper presented in 1995, Futer showed the efficiency of the time domain signaling method in detecting various box defects compared to other vibrational signal processing methods. [2] In [3], they used the Kurtosis statistical method to monitor vibrating gears. The application of this monitoring method is at the power plants. They used a few vibrational signals to study the Kurtosis benchmark, and the results showed that the Beta-Kurtosis method has a good ability to troubleshoot. In 2013, Gharavian and colleagues reviewed the troubleshooting of the car's gearbox, with many benefits to tracking the car's condition and troubleshooting it in previous related work. Jenna et al. (2014) showed that the use of active de-noise method using a reference signal obtained from a healthy gear in the design of a comparative filter based on the least squares error method, is able to improve the signal-to-noise ratio, the signal generated by defective gear. [4] In 2017, Yazdanian et al. presented a method for identifying simultaneous faults based on the non-burnt Bayesian classification method for rotating systems without using synchronous training data for training the neural network. He showed that this method, using the machine learning method and based on the Bayesian probability distribution function, has the great ability to estimate the simultaneous defects of the rotary system in comparison with the other methods proposed for troubleshooting in this case [5]. In this paper, to troubleshoot and determine the types of faults in the gearbox, the effect of each failure in four different modes and with two methods of Kurtosis and Beta-Kurtosis is performed to identify and resolve the failure and malfunction of the gearbox before use with the use of vibration analysis.

\section{Research method}

Figure (1) schematically shows the data processing process of the Pride gearbox laboratory equipment.

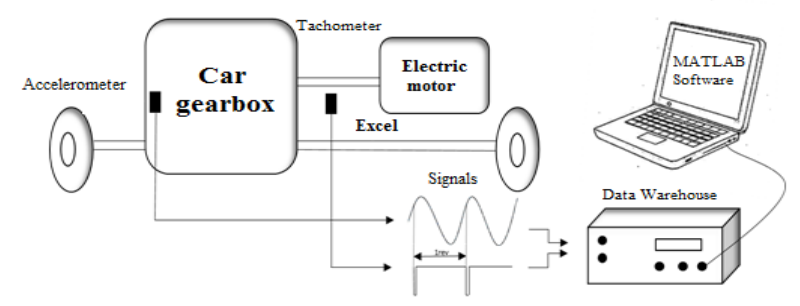

Fig. 1: Schematic Representation of the Data Processing Process of Gear Box Equipment.

Figure (2) also shows the various parts of the launch and data processing of the Pride gearbox laboratory equipment. 


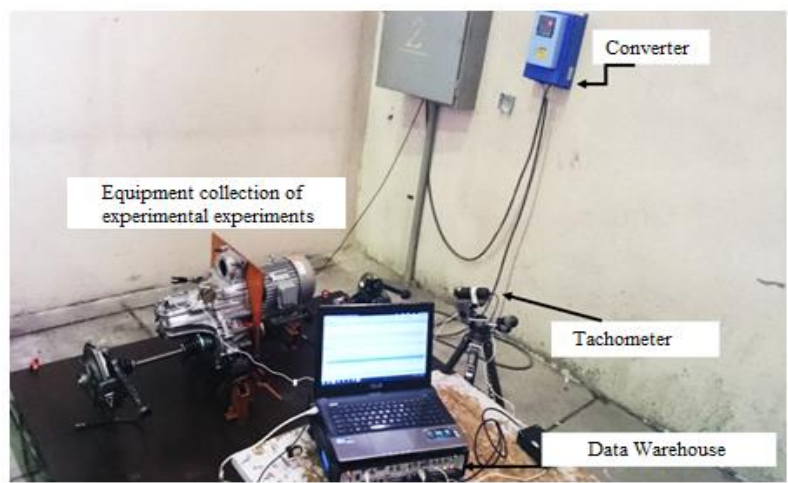

Fig. 2: Different Parts of the Set up and Data Processing of the Gearbox Equipment Laboratory.

\subsection{Defects created on the tested gearbox}

To create fault modes, once the bearings and again the gear are replaced on the shaft shown in fig. 3, and instead replace the defective part as shown in Figures 4 and 5, and install and reequip, set up and carry out data operations. It is done on it. At the end, both defective components were mounted on the equipment so that system data can be combined with the simultaneous and combined failure modes. The internal schematic of the gear box and the gear bearing and gear selected for testing are shown in Fig. 3.

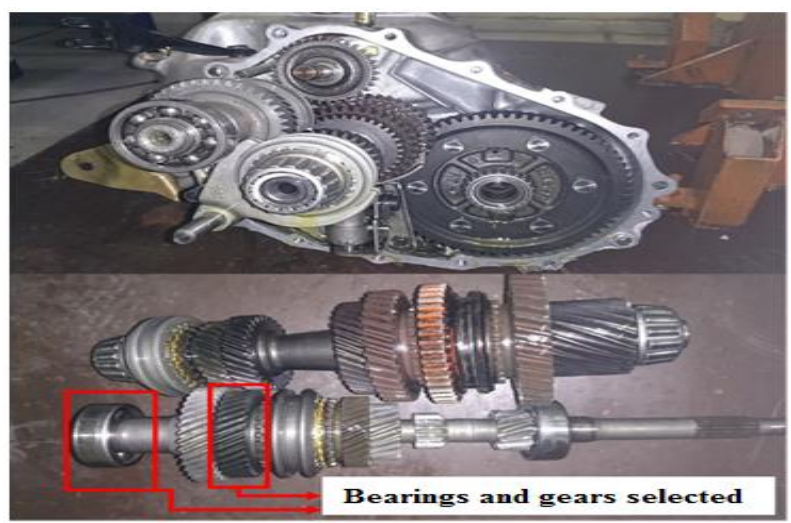

Fig. 3: Interior View of the Gearbox to Display the Selected Bearings and Gear for Testing.

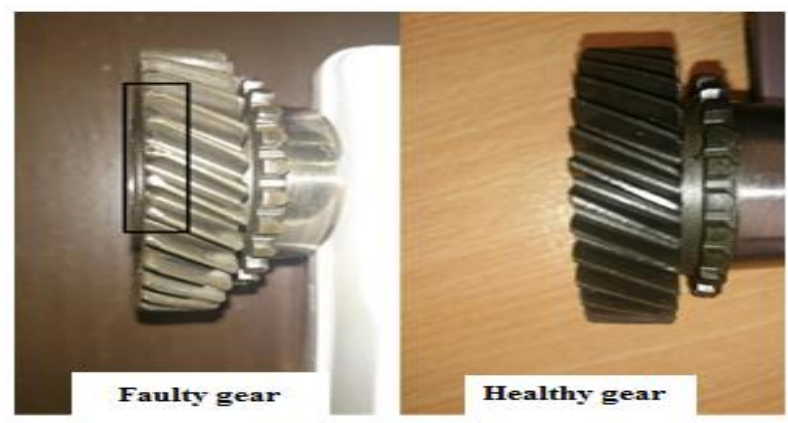

Fig. 4: Healthy Gear and Faulty Gear.

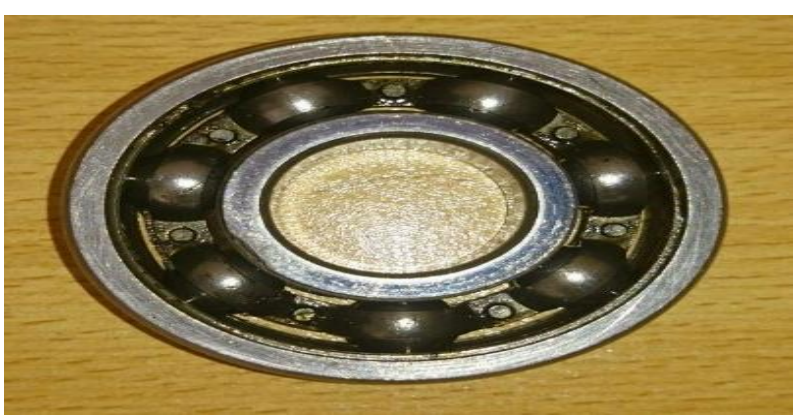

Fig. 5: The Selected Bearings for Testing.

\subsection{Method of kurtosis}

The Kurtosis method is one of the techniques used to identify impulses in the ventricle of the signal, for example, to determine the effect of the presence of a defect on ball bearings and gears. Kurtosis In fact, the fourth torque of the signal is normalized, which is determined by a value. This process, which is one of the most practical methods, includes three tools for squaring the analog signal. [3] In the mathematical expression for an X signal, the value of Kurtosis is obtained from the following integral:

$k=\frac{\int_{-\infty}^{+\infty}(x-\bar{x})^{4} p(x) d x}{\sigma^{4}}$

\subsection{Method of beta-kurtosis}

Beta-Kurtosis is the fourth torque parameter the normalized time signal based on the beta distribution. The beta distribution is a suitable statistical distribution for vibrational signals (random signals), this distribution is used to diagnose gear boxes with the help of vibrating signals. For example, consider the $u$ signal, which is normalized in range 0 and 1 . The beta probability distribution function is defined as:

$f_{B}(u)=\frac{1}{B(\alpha, \beta)} u^{\alpha-1}(1-u)^{\beta-1}$

Where in

$$
B(\alpha, \beta)=\int_{0}^{1} u^{\alpha-1}(1-u)^{\beta-1} d u
$$

In addition, $\alpha$ and $\beta$ and numerical parameters are positive. The Kurtosis benchmark is a statistical measure that can be used to diagnose and diagnose systems. This criterion can be used to determine the peakedness of the signal peaks at the same time in a statistical distribution. To determine the Kurtosis criterion, the central moment of the signal (M) must be determined. As you can see, this parameter is a statistical parameter that is derived from the beta function. This feature can be used as a criterion for vibrating signal behavior.

\section{Results}

In this section, firstly, the description of the extraction of Kurtosis and $\beta$-Kurtosis information from accelerated data measured using MATLAB software is presented and finally the results are reviewed and compared. LabVIEW SignalExpress software is used for data capture. After determining the number of accelerometers used and determining the sensitivity of each of them, as well as defining its specifications on the software, we begin to give data. The functional characteristics of the gearbox for various modes are given in Table (1).

Table 1: Test Specification for a Healthy State of Gearbox Operation

\begin{tabular}{llll}
\hline Experiment state & State & Gearbox speed (rpm) & Gear number \\
\hline 1 & Healthy & 150 & 3 \\
\hline
\end{tabular}

\subsection{Healthy gearbox results}

By extracting the signal for each round of the system, you can extract the Beta-Kurtosis attribute for each round. First, it is necessary to calculate the parameters $\alpha$ and $\beta$ and to calculate $\alpha$ and $\beta$, the mean values and the time signal variance must be calculated. In this paper, for each short signal, the mean and variance values, and subsequently the values of the parameters $\alpha$ and $\beta$, are calculated for each of the rounds above. Finally, the value of the Beta-Kurtosis parameter is obtained. In other words, the Beta-Kurtosis value is a function of a far-off num- 
ber. In Fig. 6, the values of this parameter are represented by the signal of the first mode. Also, in order to compare this criterion with Kurtosis coefficients, the value of this parameter is also calculated for different periods in MATLAB software and is shown in Fig. 7.

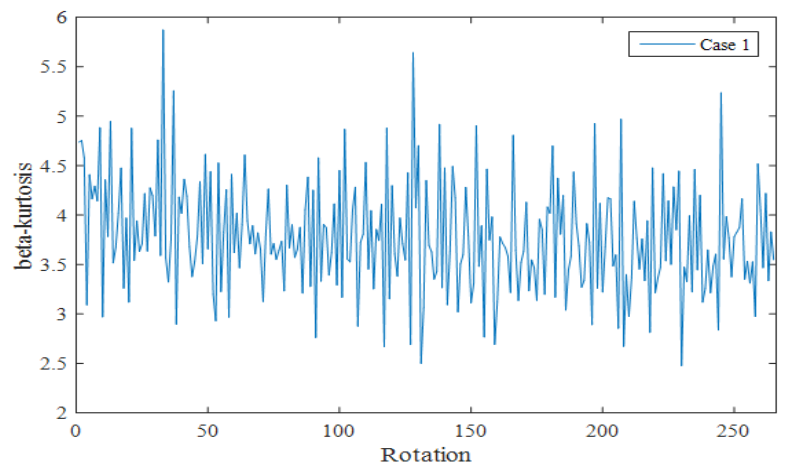

Fig. 6: Beta-Kurtosis Characteristic Diagram for Healthy State of Gearbox Operation.

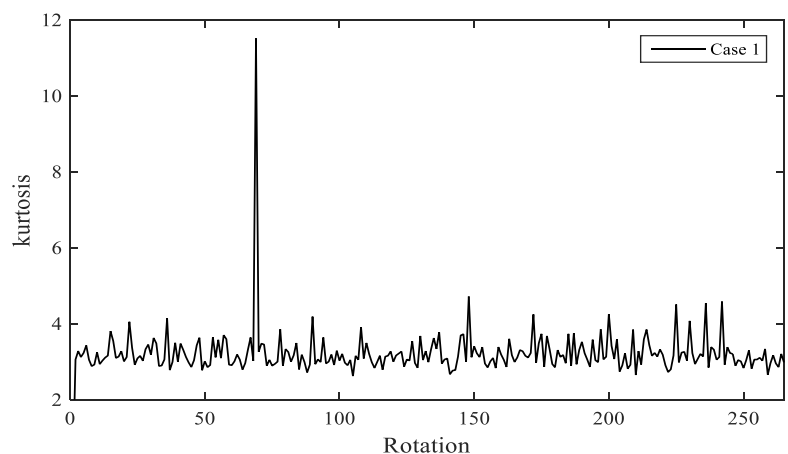

Fig. 7: Kurtosis Characteristic Diagram for Healthy State of Gearbox Operation.

As shown in Figures 6 and 7, the value of the Beta-Kurtosis and Kurtosis parameters is the far-field function, or, in other words, the time function.

\subsection{Results for the failure mode of the end of the gearbox shaft}

By extracting the signal for each round of the system, you can extract the Beta-Kurtosis attribute for each round. First, the mean values and the variance of the short-time signals are calculated, and then the parameters $\alpha$ and $\beta$ are calculated. Finally, the Beta-Kurtosis value is shown as a function of the round number in Fig. 8, corresponding to the second state signal. Also, in order to compare this criterion with Kurtosis coefficients, the value of this parameter is also calculated for different periods in MATLAB software and is shown in Fig. 9.

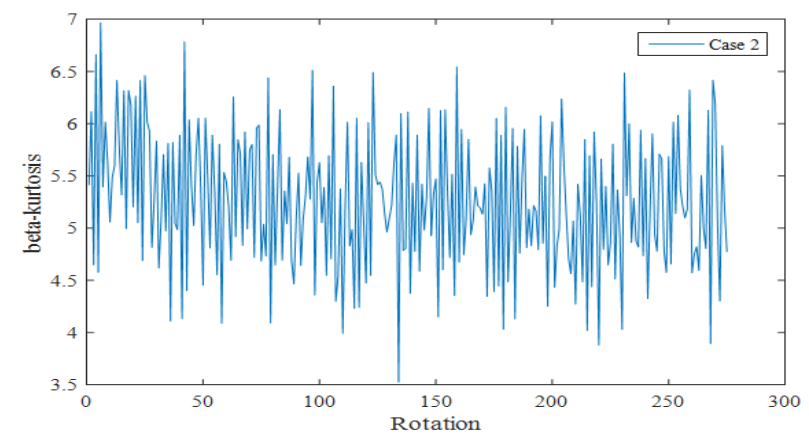

Fig. 8: Beta-Kurtosis Characteristic Diagram for the Operation of a Defective Gearbox with a Bearing on the Shaft End.

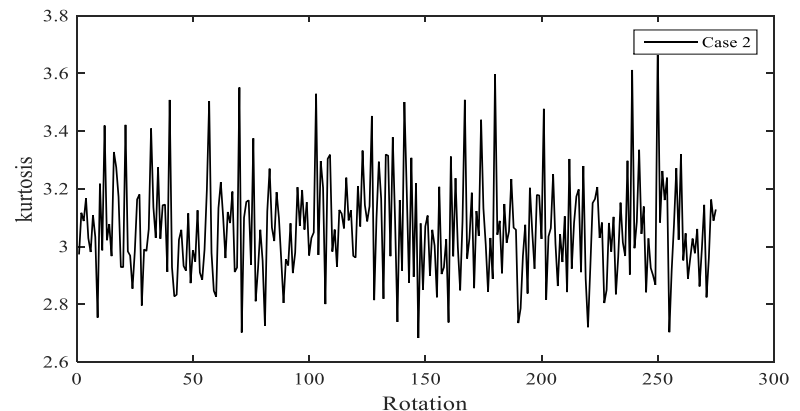

Fig. 9: Kurtosis Characteristic Diagram for the Operation of the Gearbox with a Defective Bearing at the Bottom of the Shaft.

\subsection{Results for the gear crash mode on the gearbox shaft}

By extracting the signal for each round of the system, you can extract the Beta-Kurtosis attribute for each round. First, the mean values and the variance of the short-time signals are calculated, and then the parameters $\alpha$ and $\beta$ are calculated. Finally, the Beta-Kurtosis value is shown as a function of the round number in Fig. 10, corresponding to the third-state signal. In order to compare this criterion with Kurtosis coefficients, the value of this parameter is also calculated for different periods in MATLAB software and is shown in Fig. 11.

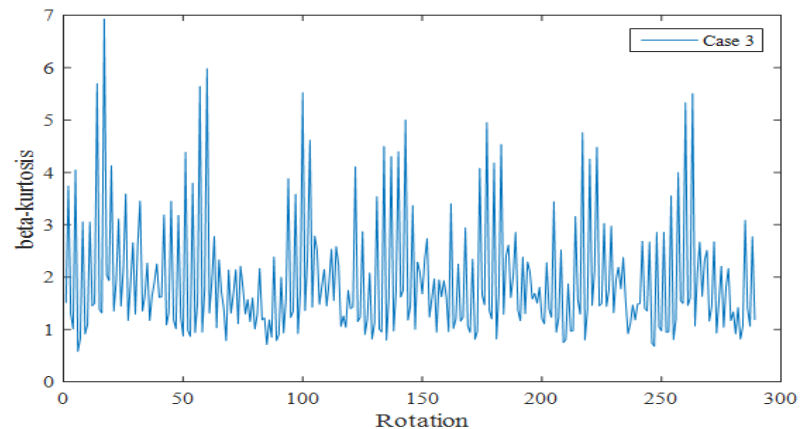

Fig. 10: Beta-Kurtosis Characteristic Diagram for Faulty Gearbox Operation Mode.

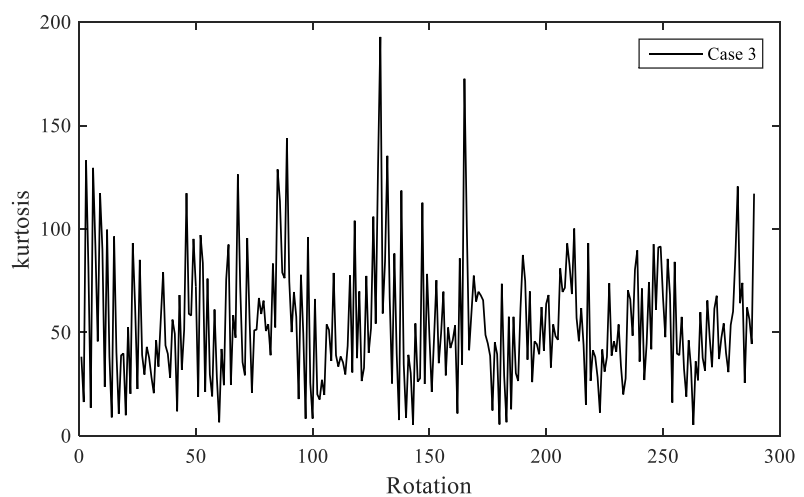

Fig. 11: Kurtosis Characteristic Diagram for the Operation of a Gearbox with a Defective Gear.

In this case, the oscillations of the values of the parameters are more severe than the previous two, and the nonparties is observed in the values of this parameter relative to the number of round.

\subsection{Results for simultaneous failure of bearings and gears on gearbox shaft}

By extracting the signal for each round of the system, you can extract the Beta-Kurtosis attribute for each round. First, the mean values and the variance of the short-time signals are calculated, and then the parameters $\alpha$ and $\beta$ are calculated. Finally, 
the Beta-Kurtosis value is shown as a function of the round number in Fig. 12, corresponding to the fourth state signal. Also, in order to compare this criterion with Kurtosis coefficients, the value of this parameter is also calculated for different periods in MATLAB software and is shown in Fig. 13.

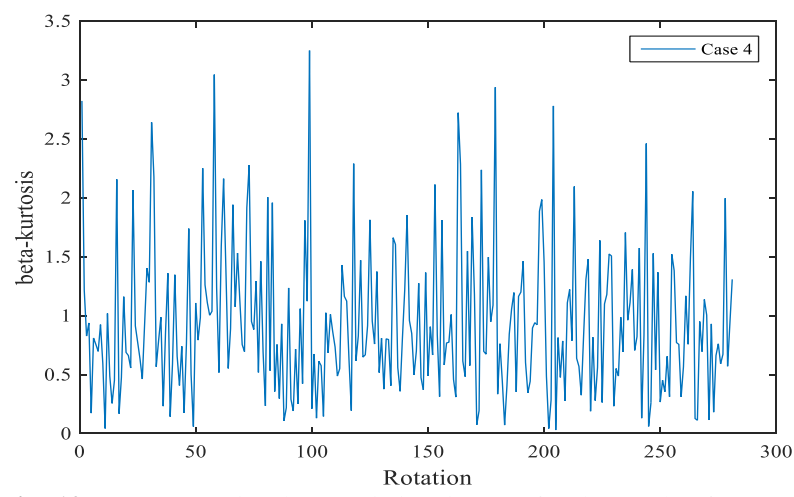

Fig. 12: Beta-Kurtosis Characteristic Diagram for the Mode of Operation of the Gearbox with the Bearing and the Defective Gear Simultaneously.

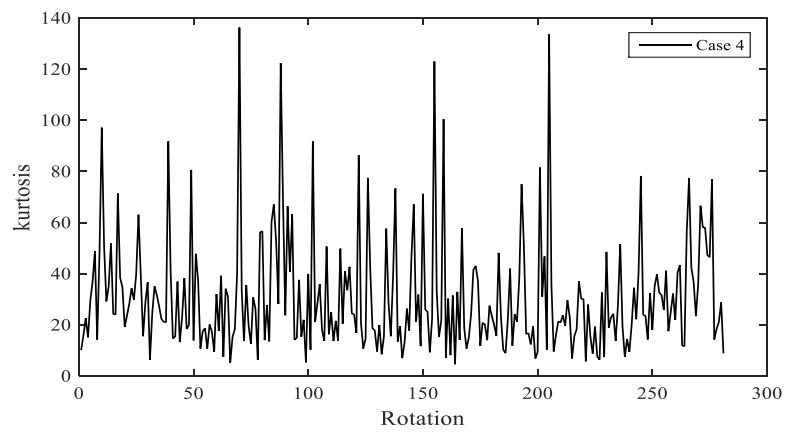

Fig. 13: Kurtosis Characteristic Diagram for the Operation of a Gearbox with a Defective Bearing and Gearbox Simultaneously.

\subsection{Results comparison}

In the above, by extracting the values of the parameters of Beta-Kurtosis and Kurtosis, the statistical characteristics for each mode were extracted. By drawing the data from the parameters and their graph in a separate form, we can clearly see the difference between different modes. Figures (14) and (15) show this difference for the four experimental modes according to Beta-Kurtosis and Kurtosis. In which case 1 is the normal operation of the gearbox, mode 2 of the gearbox with a defective bearing, the mode 3 of the gearbox with the defective gear, and the mode 4 of the gearbox with the bearing and the gear is defective simultaneously. It can be seen that, based on BetaKurtosis, all four modes can be cleaned properly, while the Kurtosis parameter is not suitable for identification and the states are not separated.

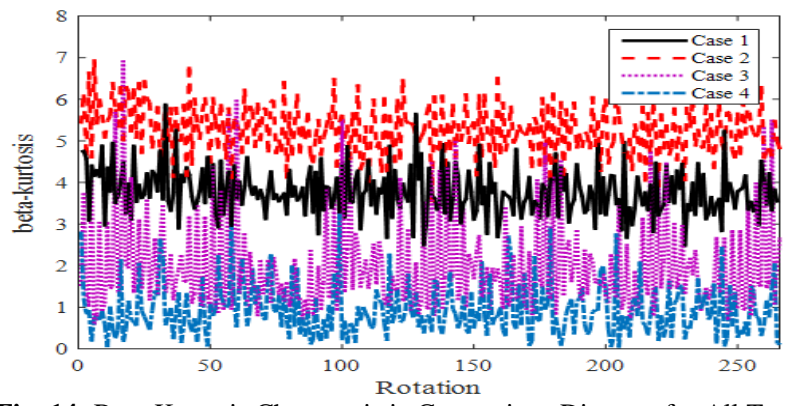

Fig. 14: Beta-Kurtosis Characteristic Comparison Diagram for All Test Modes.

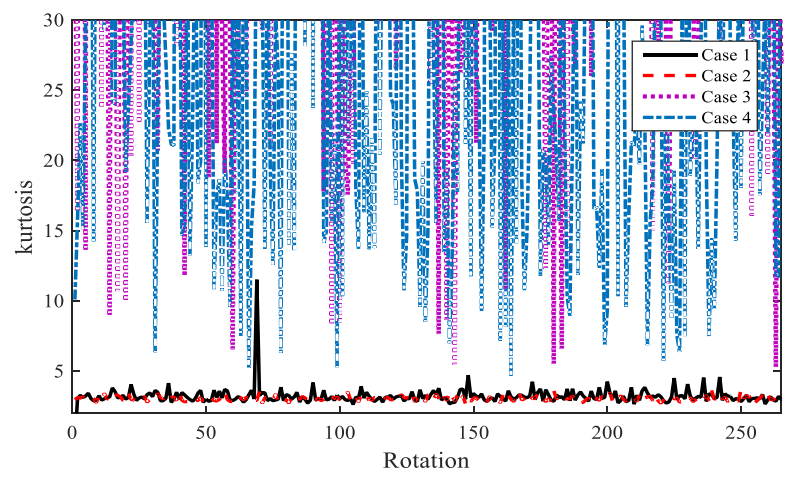

Fig. 15: Kurtosis Characteristic Comparison Diagram for All Test Modes.

Based on the above figures, the use of the Kurtosis parameter is discarded and only the Beta-Kurtosis parameter is used. It is clear from Fig. 14 that all four states are almost recognizable. Of course, in some periods, the parameter Beta-Kurtosis enters the region, especially in the third and fourth modes. This phenomenon suggests that, for accurate detection, the signal under consideration should include, in terms of the length of time, a large number of cycles, so that the average is detected correctly. In this regard, we consider the statistical characteristic of the Beta-Kurtosis parameter as a state-detection criterion. These two statistical characteristics are:

1) The mean of the Beta-Kurtosis signal (Mean)

2) The standard deviation of the Beta-Kurtosis signal

Using these two Beta-Kurtosis statistical characteristics, base signals are used as base values for defect detection, and by comparing these statistical characteristics with the statistical characteristics of the various signals generated in defective cases. The power of the type of defect created in the gearbox is detected. The values of these parameters for different test modes are shown in Fig. 16.

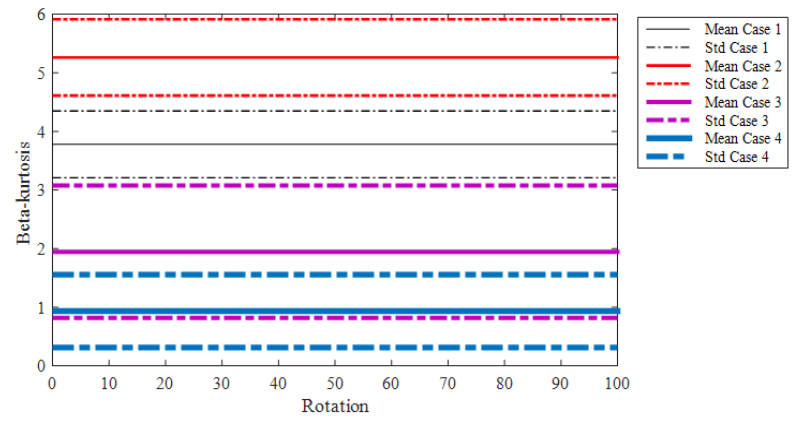

Fig. 16: Comparison of the Mean and Standard Deviation of the BetaKurtosis Characteristic for All Test Modes.

It should be noted that all experiments carried out with a distance greater than 250 were associated. In Fig. 16, the mean values and standard deviation values are obtained based on the 100 first rounds to be an educational characteristic. In the sense that using the remaining 150 residues, the ability of these attributes can be measured to detect situations.

\subsection{Detection of conditions (test method)}

In this section, we will diagnose the states based on the predetermined signal. These signals are not used in the extraction of the criteria in Fig. 19-19. The general process of work is that at first the statistical characteristics (mean and standard deviation) of the Beta-Kurtosis signal of the base signals (4 existing modes of advance) are extracted, then the Beta-Kurtosis of the existing signal is extracted and examined by the visual comparison method In which region is the base signal from the values of the Beta-Kurtosis statistics? First, the video criterion is used for this task and the test signal is taken from each of the four modes for the last 100 rounds of motion, and then the Beta- 
Kurtosis values for each mode are extracted. In the image criterion, the Beta-Kurtosis signal is used in the standard deviation interval for detection. In Figs (17) to (20), the image recognition mode is displayed for all four signals.

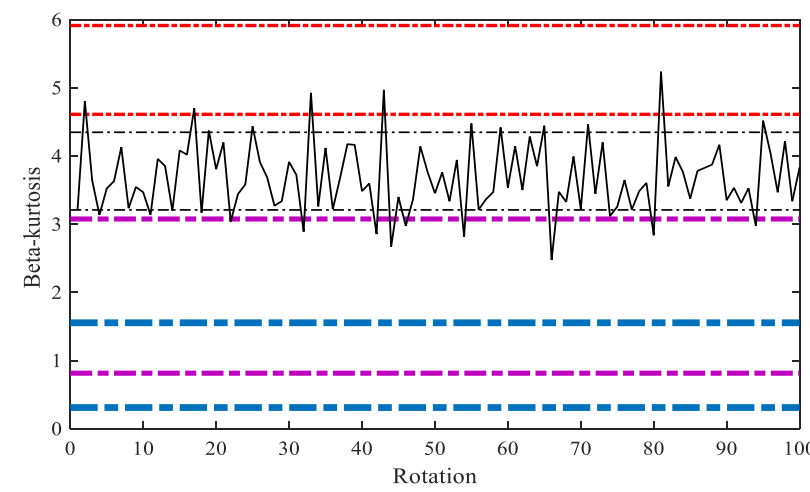

Fig. 17: First Signal Detection Test Based On Video Standard Deviation.

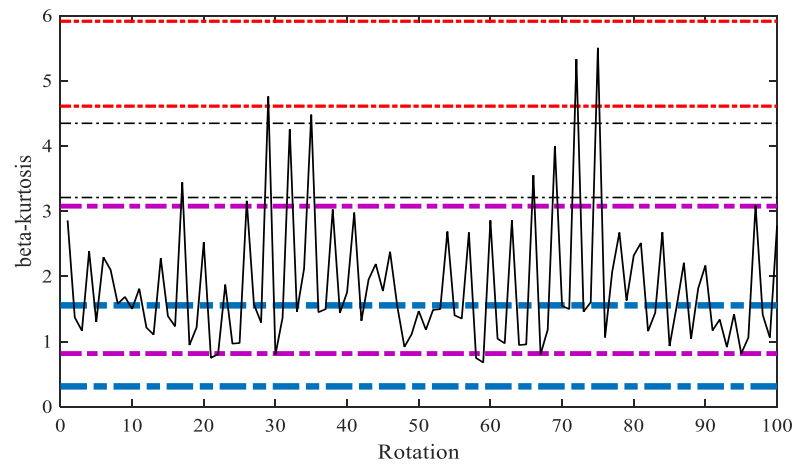

Fig. 20: Second Signal Detection Test Based on Video Standard Deviation.

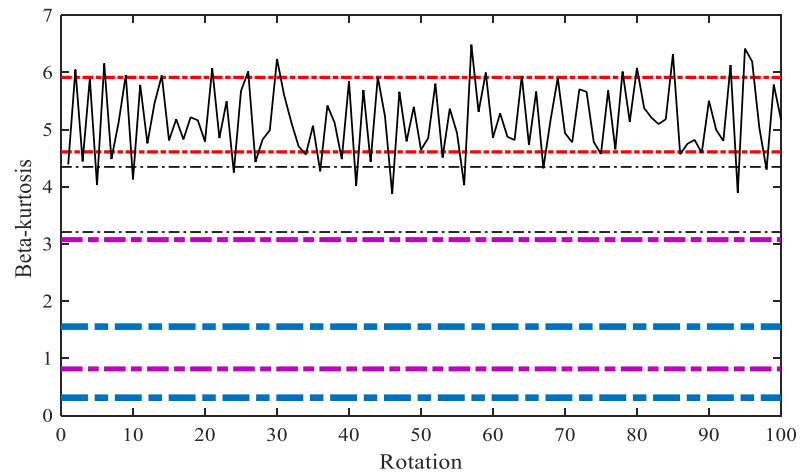

Fig. 21: Third, Signal Detection Test Based on Video Standard Deviation.

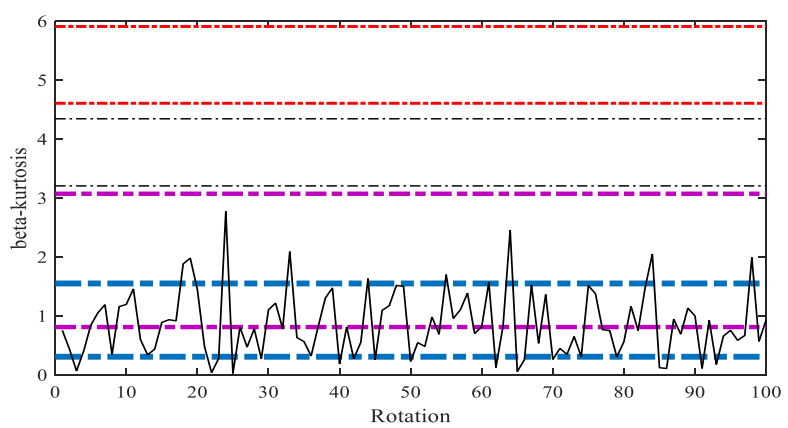

Fig. 22: Fourth Signal Detection Test Based on Video Standard Deviation.

As you can see from the four forms above, it is possible to determine the mode state based on the video criterion. In the following, an average numerical criterion is used for diagnosis. In Table 2, the mean values of the test signal are reported together with the mean values of the training, and the smallest distance from these values is considered as state detection.

Table 2: Detection of Four Pre-Determined Test Signals Based on the Mean Value of Beta-Kurtosis

\begin{tabular}{|c|c|c|c|c|c|}
\hline Signal Training Mode: & & Mode 1 & Mode 2 & Mode 3 & Mode 4 \\
\hline Average Training: & & 3.779 & 5.262 & 1.946 & 0.934 \\
\hline Signal Test Mode & Mean & Test dis & training & & \\
\hline Mode 1 & 3.682 & 0.097 & 1.580 & 1.736 & 2.748 \\
\hline Mode 2 & 5.161 & 1.382 & 0.101 & 3.215 & 4.227 \\
\hline Mode 3 & 1.843 & 1.936 & 3.416 & 0.103 & 0.909 \\
\hline Mode 4 & 0.869 & 2.910 & 4.393 & 1.077 & 0.065 \\
\hline Diagnosed: & & Mode 1 & Mode 2 & Mode 3 & Mode 4 \\
\hline
\end{tabular}

As can be seen, the process of variation of the Beta-Kurtosis criterion in different defective and healthy states is independent of each other, and each specific case has a specific Beta-Kurtosis statistical criterion, so that by varying the amplitude of the vibrations in the measured signal, the criterion value of Beta-Kurtosis has begun to change, indicating a benchmark for fault-finding. Due to the fact that this statistical method needs to be followed by the process of defect rectification, this method can be used to monitor the devices.

\section{Conclusion}

In the foreground, using the previous work done, will try to use the Beta-Kurtosis method and the provision and implementation of laboratory equipment has paid attention to the diagnosis and monitoring of the Pride transmission and examines the vibrations of a healthy and unhealthy state of operation. Of course, it should be noted that all studies for the Kurtosis method are also carried out and compared. In the end, after all the scenarios were analyzed for the operation of the gearbox as healthy or defective by the two criteria of Kurtosis and Beta-Kurtosis, it was found that, based on Beta-Kurtosis, all four modes can be properly cleaned up while the Kurtosis parameter is not suitable for detecting defects in this gearbox, and the states are not separated. It can be said that the
Beta-Kurtosis benchmark for troubleshooting the Pride's gearbox is a suitable method that can be used in the industry to monitor the gearbox of this car.

\section{References}

[1] McFadden, P. D. (1989). Interpolation techniques for time domain averaging of gear vibration. Mechanical systems and signal processing, 3 (1), 87-97.

[2] Futter, DN (1995). Techniques for monitoring the large gearboxes in the power industry. Insight, 37 (8), 591-594.

[3] Wuxing, L., Peter, W. T., Guicai, Z. \& Tielin, S. (2004). Classification of gear faults using cumulants and the radial base function network. Mechanical systems and signal processing, 18 (2), 381-389.

[4] Yena, D.P., Sahoo, S., \& Panigrahi, S.N. (2014). Gear fault diagnosis using active noise reduction and adaptive wavelet transform. Measurement 47: 356-372.

[5] Asr, M. Y., Ettefagh, M. M., Hassannejad, R., \& Razavi, S. N. (2017). Diagnosis of combined faults in Rotary Machines by NonNaive Bayesian approach. Mechanical Systems and Signal Processing, 85, 56-70.

[6] Andrew D. Dimarogon and Sam Haddad. (1992), "Vibration for Engineers." Machinary Vibration: Monitoring and Diagnosis 14,675-706. 